\title{
Immunoterapia del cancro
}

I due articoli pubblicati in questo numero speciale di Global \& Regional Health Technology Assessment affrontano una problematica di straordinaria importanza: la difficile impresa di mantenere sostenibile il nostro Sistema Sanitario, che resta, malgrado alcune vicissitudini, un esempio a livello mondiale, perché basato su principi di universalità ed equità (tra l'altro ben presenti nell'art. 62 della nostra Costituzione). In effetti, dopo qualche anno di apparente "stallo", l'industria farmaceutica (e la ricerca pubblica, della quale non può essere dimenticato il contributo sostanziale) ha tirato fuori dal cappello una serie di terapie innovative che fanno intravedere cambiamenti epocali nel trattamento di numerose malattie croniche. In realtà, una serie di elementi importanti, tra i quali il cambiamento demografico e l'invecchiamento della popolazione, l'arrivo di straordinarie innovazioni bio-mediche (non soltanto farmacologiche), la nascita della medicina personalizzata (ma anche, purtroppo, della medicina difensiva) fanno intravedere un futuro nel quale la spesa per la salute è destinata a una crescita esponenziale.

Ma, al di là degli aspetti farmaco-economici, è importante parlare anche dei farmaci che hanno innescato la discussione. E di vera innovatività. E di vera innovatività, che, insieme all'appropriatezza, è l'elemento che dovrebbe guidare l'allocazione delle risorse disponibili. La Food and Drug Administration (FDA) e recentemente anche l'European Medicine Agency (EMA) hanno coniato il termine di breakthrough drugs, per significare la differenza che esiste con numerosi altri farmaci, pur innovativi, che però non sono in grado di modificare in modo sostanziale la storia naturale di una malattia e l'aspettativa di vita dei pazienti.

Un esempio recente è l'introduzione dei Direct Acting Antivirals (DAA) per la cura dell'infezione da virus dell'epatite C: una straordinaria innovazione, in grado di guarire dall'infezione e in parte di arrestare la progressione della malattia epatica, che tuttavia deve confrontarsi - come ai tempi dei farmaci antiretrovirali per l'HIV - con problemi di "costi" e di accesso: a parte i "prezzi" proibitivi di questi nuovi farmaci (un elemento affrontato molto bene dall'AIFA in termini negoziali: i prezzi caleranno, soprattutto perché arrivano a breve nuove combinazioni rispetto alle esistenti), i problemi sono soprattutto strategici: trattare tutti gli infetti (con l'opportunità di "eradicare" l'infezione dal Paese) o i pazienti più gravi? Quali i costi della malattia residuale, nel caso si scegliesse la seconda opzione? Tra quanti anni saranno evidenti i risparmi a fronte di una spesa "immediata"?

Un discorso a parte meritano i (costosi) farmaci biologici, gli anticorpi monoclonali, che sono stati sviluppati (e registrati) per la terapia di alcuni tumori, e di alcune malattie su base immunologica.

In realtà, la storia dell'uso degli anticorpi monoclonali (Mab) in ambito oncologico è lunga e di straordinario successo, ed è basata sulla capacità dei Mab di mantenere le preziose specifiche immunocompetenze degli anticorpi naturali, e di lavorare come farmaci "intelligenti" capaci di riconoscere selettivamente un bersaglio molecolare di interesse clinico, ed eventualmente di veicolare su di esso, direttamente (cioè attraverso una loro intrinseca attività) o indirettamente (quando vengono coniugati ad altri composti chimici/biologici) una qualche azione farmacologicamente rilevante. In campo diagnostico, la validità degli anticorpi è ormai solidamente riconosciuta: un anticorpo specifico per un antigene tumoreassociato coniugato con un tracciante radioattivo specifico, rende possibile l'identificazione della massa tumorale e l'individuazione di focolai metastatici con una sorprendente sensibilità. Ma è il settore terapeutico quello che ha fatto registrare i maggiori progressi nell'ultimo decennio: la capacità dei Mab di riconoscere selettivamente una cellula neoplastica (a condizione, ovviamente, che questa esprima il relativo recettore) ne favorisce l'eliminazione o distruzione, attivando uno dei meccanismi naturalmente mediati dalle immunoglobuline (apoptosi, CDC, ADCC) oppure "accompagnando" sul bersaglio cellulare una molecola (farmaco di sintesi, radionuclide, tossina), o un componente del sistema immune (celIula immune) con attività citotossica. È il caso del rituximab uno dei primi anticorpi monoclonali a essere approvato per uso clinico, utilizzato nel trattamento del linfoma non-Hodgkin, della leucemia linfocitica cronica e in talune malattie autoimmuni. In altri casi, gli anticorpi monoclonali possono essere "armati", coniugandoli a molecole che ne potenziano l'efficacia terapeutica: la specificità di azione rimane legata alla proprietà dell'anticorpo, mentre l'azione farmacologica contro la cellula target viene per lo più affidata a una componente non anticorpale. È il caso del trastuzumab, recentemente approvato per il trattamento dei tumori mammari metastatici esprimenti l'antigene HER2 (Human Epidermal Growth Factor Receptor 2), che viene coniugato a un agente antimitotico il cui effetto antiproliferativo e citotossico viene prevalentemente mirato sulle cellule neoplastiche che l'anticorpo è in grado di intercettare. 
Ma è a una nuova classe di anticorpi monoclonali terapeutici che vale la pena di accennare: gli immune-check points inhibitors, cioè quelle molecole anticorpali che permettono di by-passare i posti di blocco che il sistema immunitario ha evoluzionisticamente sviluppato per difendersi da una strabordante presenza di antigeni. Farmaci che promettono di aprire straordinari orizzonti, per il trattamento dei tumori.

Dopo una decade di fallimenti delle strategie (al punto che si erano perse le speranze) per indurre il sistema immunitario ad attaccare le cellule tumorali, la scoperta dei meccanismi patogenetici alla base della cosiddetta exhaustion della risposta immune (e non solo contro i tumori) hanno portato alla sintesi di farmaci in grado di riaccendere i linfociti T. Gli immune-check points sono purtroppo numerosi, e il futuro sarà di attaccarli in modo combinato, con diversi Mab e insieme ai nuovi farmaci chimici antineoplastici. Nel frattempo, si è lavorato su uno di questi, il recettore PD-1. Con i primi farmaci (nivolumab, pembrolizumab) sono state osservate remissioni importanti (in alcuni casi anche "guarigioni") di persone con forme resistenti al trattamento classico di melanomi, leucemie, linfomi, e alcuni tumori solidi avanzati: si tratta di aumenti di aspettativa di vita mediana estremamente significativi, se rapportata ai piccoli passi "chemioterapici" degli ultimi vent'anni. Insomma, siamo di fronte a una rivoluzione "concettuale" straordinaria: non solo si vanno ad attaccare le cellule neoplastiche, ma abbiamo capito come attivare il nostro principale alleato, il sistema immunitario.

Stefano Vella

Direttore, Dipartimento del Farmaco, Istituto Superiore di Sanità, Roma 